\title{
Fixation of Unstable Peritrochanteric Fractures of Femur Using Gamma Nail
}

\author{
Mohammed Al Zahhar ${ }^{a}$, Emad Esmat ${ }^{a}$, Amr El Gazzar ${ }^{a}$, Basem Arnaout ${ }^{\text {b }}$
}

a Department of Orthopedic surgery, Faculty of Medicine Benha University, Egypt. b Department of Orthopedic surgery, Al Ahrar teaching hospital, Egypt.

Correspondence to: Basem Arnaout, Department of Orthopedic surgery, Al Ahrar teaching hospital, Egypt.

\section{Email:}

basemmamdouh11@hotmail.com

Received: 22 July 2019

Accepted: 2 July 2021

\begin{abstract}
:
Introduction:The early operative treatment of the peritrochanteric fractures is widely accepted practice and different sliding nail-plate systems, including Gamma Nails (GN) are used in the treatment. The GN was designed to treat unstable trochanteric fractures. Aim: The aim of this study was to discuss the results of treatment of unstable peritrtrochanteric fracture of femur in 30 patients treated by fixation by Gamma nail regarding its outcome. Patients and methods: The study was based on a total of 30 patients surgically treated for the unstable peritrochanteric femoral fracture in the period between August / 2014 till July /2017. All patients underwent gamma nail osteosynthesis. The average age of the patients was 67.2 years (range 40-81 years) with 13 male patients and 17 female patients. The average operative time was $71.8 \mathrm{~min}$ (range 45 -100 min). Clinical and radiographic assessments were performed during follow-up visits at 2 weeks, 6 weeks, 12 weeks and 6 months up to one year. Results:
\end{abstract} According to the final Harris hip score, results were excellent in 7 patients, good in 13 patients, fair in 5 patients and poor in 4 patients while one patient ends with deep infection and nonunion.Conclusion: This study suggests that the gamma nail is an excellent alternative in treatment of unstable peritrochanteric fracture as it gives advantage of closed technique, allows early mobilization and early weight-bearing. It involves lesser perioperative complications.

Key words: peritrochanteric, Gamma nail, osteosynthesis

\section{Introduction:}

Hip fractures can occur at any age; however, they most commonly occur in the elderly.
The elderly are most vulnerable to hip fracture complications as they are the ones 
who often present with associated comorbidities (1) .The incidence of the more unstable, comminuted fractures is increasing, paralleling the increased longevity of the world's population (2).

Increased surgical complexity are associated with unstable fracture patterns. The most important aspect of any classification is the determination of fracture stability which is provided by the presence of an intact posteromedial cortical buttress (3).

The primary principle in the treatment of intertrochanteric hip fractures is to reestablish the continuity of bone between the head and neck fragment and the shaft and to place the fixation device central in the femoral head. This will allow the bone to carry the majority of the load transmitted across the hip, minimizing the risk for failure, and allowing frail patients to be mobilized early with sufficient weight bearing so that they can gain a reasonable degree of independence. (4).

Intramedullary implants have revolutionized the management of trochanter fracture, as they carry all possible advantage over their counterparts, extramedullary implants. They are inserted with minimal soft tissue injury technique, less blood loss, less limb shortening and less decrease in medial offset so allows for faster rehabilitation (5).

Intramedullary fixation devices, which combine a hip screw with either a short or long intramedullary nail such as the Gamma nail, have the theoretical advantages of percutaneous insertion, a lower bending moment on the fixation device, and an intramedullary buttress that precludes excessive medial migration of the shaft (6).

Therefore, this study was designed to discuss the results of treatment of unstable peritrtrochanteric fracture of femur in 30 patients treated by fixation by Gamma nail.

\section{Patients and methods:}

The study was conducted on a total of 30 patients surgically treated for the unstable peritrochanteric femoral fracture in the period between August / 2014 till July /2017. All patients underwent gamma nail osteosynthesis.

The study was approved by Banha scientific ethical committee. All the 30 cases were planned for follow-up examination for a period of one year from the date of operation. Full workup, including age, sex, medical history, type of fracture, mechanism of injury, and plain radiographs was performed on admission. 
- Inclusion criteria:

The study included patients with unstable intertrochanteric fractures involving the calcar. Unstable fractures in this study are those involving the posteromedial cortex, those with 3 or 4 parts comminution, those with subtrochanteric extension fractures with reverse oblique pattern. The study also included patients aged more than 16 years old after closure of the physis of the greater trochanter.

\section{- Exclusion criteria:}

Patients with stable peritrochanteric fracture with intact calcar, previous femoral deformity (congenital or traumatic), those aged less than 16 years with open greater trochanteric physis \& elderly with multiple co-morbidities like terminal stages of malignancy, patients with severe heart \&/or respiratory failure that interfere with anesthesia were excluded from the study.

\section{- Preoperative assessment:}

The pre-operative parameters that were recorded included the age that ranged from 40 to 81 years with the mean age about 67.2 years and sex which was 13 male \& 17 female, the side of the fracture was 12 fractures involving the right femur \& 18 fractures involving the left side while the pattern of fracture was $82 \%$ of type A2 \& A3 while the remaining $18 \%$ is type A1.3 according to AO classification (diagram 1).

Pre-operative assessment of the medical history showed that 12 patients were medically free, 10 patients were hypertensive, 9 patients were diabetic, 3 patients were cardiac \& one patient was hepatic. Preoperative assessment included also previous surgery, any allergy \& femoral deformity.

Plain X-rays were obtained on admission, and all fractures were classified

according to the AO classification.

Post-operative care:

Physiotherapy, mobilization from bed and partial weight bearing were allowed for patient with 3 parts fragment fracture \& minimal comminution with sufficient bone stock \& stable fixation or just toe touch for those patients with highly comminuted fractures \& poor bone stock.

Average follow up in the clinic was 12 months on basis of two, six weeks, then after three, six \& twelve months from operation. The patients were examined clinically and radiologically at each visit. Six to eight weeks after surgery, full weight bearing was allowed according to healing process \& 
appearance of callus in x-rays.

Clinical examination was directed at checking the pain, tenderness, range of motion, walking with support, sitting, wearing shoes, deformity and stairs that were the parameters of Harris hip score (7). Other parameters like hotness, redness, and wound discharge as signs of infection were also recognized. A scoring system below 70 is considered poor, $70-79$ is considered fair, 80 - 89 is considered good, while a score 90 100 is considered excellent (8).

\section{Results:}

The current study included 13 males and 17 females. The right side was affected in 12 patients and the left side in 18 patients. The age ranged from (40 to 81 years) with the mean age is 67.2 years old (table 1). The mechanism of injury was fall in 20 patients \& RTA in 10 patients. The patients stayed in the hospital for about (4-7 days) with the mean hospital stay are 5 days.

There were 27 patients (90\%) with good reductions while there were 3 cases with mild displacement $(<8 \mathrm{~mm})$ in both AP \& lateral views but considered accepted. The fracture had healed in all the patients with no significant varus displacement except in 3 patients where their fracture had healed with mild <20 degrees varus angulations. Lag screw position (diagram 2) was central in anteroposterior \& lateral views in 15 cases $(50 \%)$ of cases while it was central in AP but posterior in lateral view in 9 cases $(30 \%)$. Lag screw was central in AP but anterior in lateral view in 2 cases $(7 \%)$ of patients. Also lag screw was inferior posterior in 2 case (7\%), while it was superior \& posterior in one case $(3 \%) \&$ it was inferior \& anterior in 1 case $(3 \%)$. There was one case reported with cutout of the lag screw through the femoral head. The Gamma nail requires short incision $(4-7 \mathrm{~cm})$. The blood loss ranged from $(120-270 \mathrm{ml})$ with the mean blood loss was $220 \mathrm{ml}$. The operative time ranged from $(45-100 \mathrm{~min})$ with the mean operative time was 71.8 minutes (Table 2). The average time of c-arm exposure was 3 minutes as it ranged from (2 - 4 min.) (Table 2). One distal locking screw was inserted in all the patients.

There were no recorded intraoperative complications like femoral shaft fractures or neurovascular injury or major bleeding in the present study. Also, there was no systemic complication from the anesthesia \& all patients recovered from the anesthesia. The mean hospital stay for the patients was 5.1 days (average 4-7 days). The time to union of the fracture ranged from 8 to 13 week with the mean time to union was 10.1 weeks. 
About 2 patients were united in 13 weeks(7\%), 4 patients was united in 12 weeks (13\%),12 patients was united in 11 weeks (40\%), 4 patients was united in 10 weeks $(13 \%), 3$ patient was united in 9 weeks $(10 \%) \& 4$ patients was united in 8 weeks (14\%).Only one patient was not united (3\%) ( Table 2). Union was assessed clinically by examining the patients for any local tenderness, limping, pain during range of motion or inability to bear weight over the affected extremity.

Also, union was assessed radiologically by appearance of callus on X-rays. 29 patients showed good \& complete union (97\%) while one patient only complicated by non-union $\&$ screw cut out (3\%).

Post-operative complications in this study include 4 cases of superficial infection without affecting fixation stability and one case of deep infection ending with non-union and screw cutout.

The final Harris hip score was excellent in 7 patients (23\%) as it ranged between (90-100) while it was good in 13 patients (44\%) as it ranged between (80-89). It was fair in 5 patients (17\%) as it ranged (70-79) \& it was poor in 4 patients $(13 \%)$ as it ranged less than 70 (diagram 3). One patient ends with deep infection and non-union (3\%).

Table 1: Data of the 30 patients with trochanteric fracture

- $\operatorname{Sex}(\mathrm{M}: \mathrm{F})$

- $\quad$ Age (mean, years)

- Fracture pattern:

Unstable (n) AO:
A 3
12
A 2
13
A1.3
5
12: 18

- Affected side

$\mathrm{Rt}: \mathrm{Lt}$
$13: 17$

67.2 
Table 2: Operative and postoperative data

\begin{tabular}{lll}
\hline Data & Range & Mean \\
\hline Operative time (means, minutes) & $45-100 \mathrm{~min}$ & 71.8 \\
Blood loss (mean, ml) & $120-270 \mathrm{ml}$ & 220 \\
Radiological exposure (mean, minutes) & $2-4 \mathrm{~min}$ & 3 \\
Hospital stay (days) & $4-7$ days & 5.1 \\
Time to union(mean, weeks) & $8-13$ weeks & 10.1 \\
Screw cutout & & 1 \\
Infection & & 4 \\
\hline
\end{tabular}

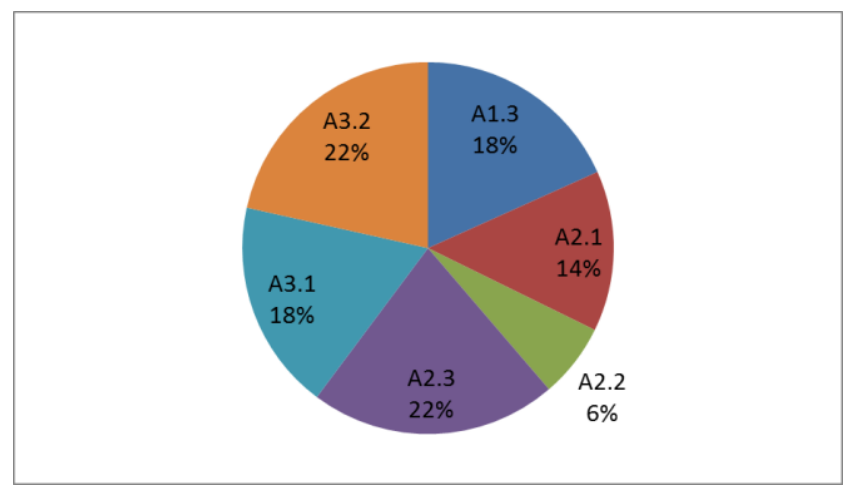

Diagram 1: showing percentage of fracture pattern

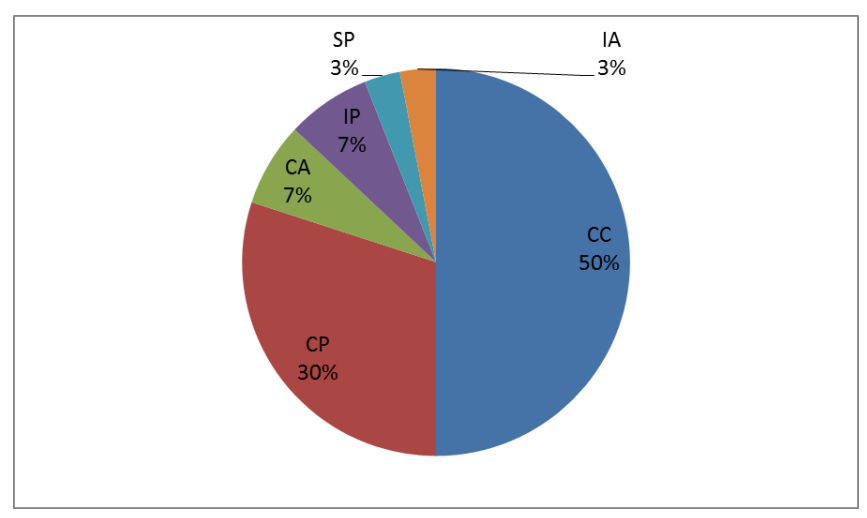

Diagram 2 : showing percentage of lag screw position 


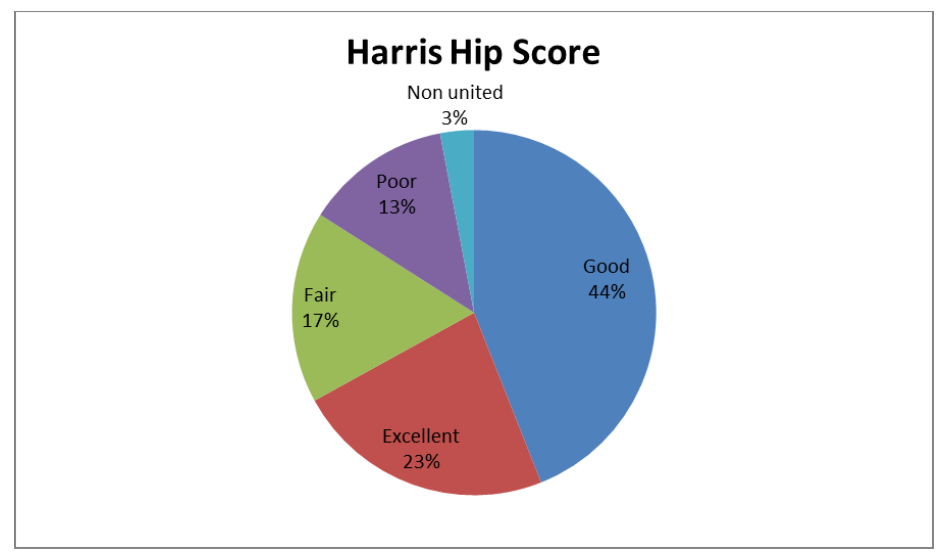

Diagram 3: showing percentage of Harris hip score outcome

\section{Discussion:}

Peritrochanteric fractures are one of the most commonly encountered fractures in clinical practice and can account for nearly $40 \%$ of admissions in most orthopedic wards. These fractures occur predominantly in people over 60 years of age and are 3-4 times more common in women than in men (9).

About $50 \%$ of these fractures are unstable. The results of management of unstable fractures are less reliable and have a high failure rate of $8-25 \%$. One goal of operative treatment is strong, stable fixation of the fracture fragments. Unstable fractures are technically much more challenging to manage than stable fractures. A stable reduction of this type of fractures requires providing medial and posterior cortical contact between the major proximal and distal fragments to resist varus and posterior displacing forces (9).

In the present study Gamma nail was applied for 30 patients (13 males, 17 females) with unstable peritrochenteric fracture femur. The mechanism of causative trauma of these patients was mostly low energy trauma in relation to bone morphology of old aged group. Operative and post-operative data included operative time, blood loss, radiological exposure, hospital stay, time to union, complications either peri or post-operative.

Pain (as one of the parameters of Harris hip score) in the mid portion of the thigh was the most common complaint with the use of gamma nail in the present study. It was recorded by Leung and his Co-workers at 
1992 that this complaint is also constant and commonly observed in other studies (10).

The operating time in this study ranged from 45 to 100 minutes and this was compared to operative time for fractures treated by GN by Leung et al. and Sharma et al. which was 40 minutes (11). Also, this study was compared to Kempf in relation to his operative time which was 41 minutes (12).

The blood loss in our patients $(220 \mathrm{ml}$ in average) was also compared to other reports (10). In the study was performed with Sharma et al. the blood loss average was 250 $\mathrm{ml}$ (11). The average blood loss with Kempf was lower $(148 \mathrm{ml}$.) in comparing with our study (12).

Functional assessment of the operated hip joint according to the Harris scale indicates a predominance of excellent and good results in unstable peritrochanteric fractures.

Radiological exposure during operation in this study was average 3 minutes which was compared with other studies. In Utrilla study, it was around 2.2 minutes (13) while exposure in Schupfner 's study was almost 3 minutes (14). It was compared to Leung and Sharma studies that exposure was less (11).

The average time of fracture healing in this study was 10.1 weeks in comparing with other studies. Average union time according to Kempf study was 11 weeks (12) that was close to Huang' s study in which union time average was 14 weeks (15) while it was 16 weeks in the study done by Lei Zhang (16).

The time to union and union rate were close for most of the studies, which confirms a widely known fact that trochanteric fractures have an excellent union potential (12).

The average lengths of hospital stay in this study (average 4 to 7 days) were definitely shorter in than those reported in the metaanalysis of 10 trials on Gamma nailing by Parker and Pryor in 1996 that was average 12 to 37 days (17) and average 10 days in Sharma study (11). Patients in Lei Zhang's study stayed in the hospital around 8.5 days (16).

In this study, no cases of intraoperative complications (femoral fracture, failed distal locking or breakage or nail jamming) were encountered during the operation in relation to other studies that showed higher incidence like Sharma (11) and Kempf study (12) which showed intra operative femoral fracture.

This study had 4 cases of superficial infection post-operative in relation to only one patient in the study by Sharma which 
was much less than in the study by Leung (11). This was also comparing with Kempf study which reveals 3 superficial infections (12). In the study of Shipper, 21 cases of superficial infection were encountered postoperative (18).

One case of screw cutout following deep infection was in our study comparable with Sharma (one patient) (11) and Leung (2 patients) (10). Also, study done by Bernard only 2 cases had screw cutout (19). Kepmf showed 6 cases of cutout in his study (12).

In this study, $90 \%$ of cases showed good reduction with $10 \%$ showed mild displacement in comparing with results of Kimpf that showed $72 \%$ with good reduction, accepted reduction in $20 \%$ of cases while poor reduction in rest of cases. (12).

Final Harris Hip Score in this study was as excellent in $23 \%$ of cases, good in $13 \%$ of cases, fair in $17 \%$ of cases and finally poor in $13 \%$ of cases. That was comparable to Sharma study that showed excellent result in $46.3 \%$ of cases, good in 36.5 of cases, fair in 14.6 of cases while poor in 2.4 of cases (11).

\section{Conclusion:}

Our study depends on using Gamma nail in fixation of unstable peritrochenteric fractures which mostly in relation of the configuration of these types of fractures were in elderly age group. This study was conducted on 30 patients with mostly low energy trauma. Following these patients during their management protocol reveals the advantages of this device in managing these fractures type.

A reduced percentage of poor outcomes, especially in unstable peritrochanteric fractures encourages the promotion of intramedullary gamma nail osteosynthesis. The final Harris hip score at 1year was excellent \& good in 20 patients $(67 \%)$ as it ranged between (80-100), while It was fair in 5 patients $(17 \%)$ as it ranged (70-79) \& it was poor in 5 patients $(16 \%)$ as it ranged less than 70 .

Gamma nail gives advantage of closed technique, allows early mobilization and early weight-bearing. It involves lesser perioperative complications. Rehabilitation is easier with this device. Proximal lag screw insertion allows dynamic osteosynthesis at fracture site. 
Weaknesses of this study include a short follow-up period and the inclusion of a small study group; however, we expect to report our long-term results in the near future.

Finally, we recommend with the previous data that the gamma nail is an excellent alternative in treatment of unstable peritrochanteric fractures.

\section{References:}

1. Shivji FS1, Green VL, Forward DP. Anatomy, classification and treatment of intracapsular hip fractures. Br J Hosp Med (Lond). 2015 May;76(5):290-5.

2. Giggl Udén \& Bo Nilsson. Hip fracture frequent in hospital. Acta Orthopaedica Scandinavica 1986, 57:5, 428-430 .

3. Thomas A. Russell. Intertrochanteric Fractures: Rockwood And Green's fractures in adults, 7th edition Copyright $\hat{A} \odot 2010$ Lippincott Williams \& Wilkins; p.1597-1638.

4. Frankel VH, Egol KA, Koval KJ, Kummer F "Stress fractures of the femoral neck". Clin Orthop Relat Res. 1998 Mar;(348):72-8.

5. Damien P. Byrne, Kevin J. Mulhall and Joseph F. Baker Anatomy \& Biomechanics of the Hip. The Open Sports Medicine Journal, 2010, 4, 5157.

6. Rosenblum SF, Zuckerman JD, Kummer FJ, Tam BS. A Biomechanical Evaluation of the Gamma Nail. J Bone Joint Surg Br. 1992 May;74(3):352-7.
7. Harris WH. Traumatic arthritis of the hip after dislocation and acetabular fractures: treatment by mold arthroplasty. An end-result study using a new method of result evaluation. J Bone Joint Surg Am. 1969 Jun;51(4):737-55.

8. Marchetti P, Binazzi R, Vaccari V, Girolami M, Morici F, Impallomeni C, Commessatti $\mathrm{M}$, Silvello L. Long-term results with cementless Fitek (or Fitmore) cups. J Arthroplasty. 2005 Sep;20(6):730-7.

9. Sharma Hemant, Deepinder Singh Loomba : Comparison of outcome of management of unstable pertrochanteric femoral fractures with dynamic hip screw and proximal femoral nail. Afr J Trauma . 2015. (4) 21-26.

10. Leung KS, So WS, Shen WY, Hui PW. Gamma nail and dynamic hip screws for peritrochanteric fractures:. A randomized prospective study in elderly patients. J Bone Joint Surg Br. 1992 May;74(3):345-51.

11. Vipin Sharma, Sushrut Babhulkar, and Sudhir Babhulkar. Role of gamma nail in management of pertrochanteric fractures of femur. Indian $\mathbf{J}$ Orthop. 2008 Apr-Jun; 42(2): 212-216.

12. Kempf I, Taglang G. The gamma nail historical background. Osteo Trauma Care $2005 ; 13: 2-6$

13. Utrilla AL, Reig JS, Muñoz FM, Tufanisco CB. Trochanteric gamma nail and compression hip screw for trochanteric fractures: a randomized, prospective, comparative study in 210 elderly patients with a new design of the gamma nail. J Orthop Trauma. 2005 Apr;19(4):229-33.

14. Schupfner R, Käsmann LT, Wagner W, Schulz AP. Complications in Treatment of 31-A Fractures with Trochanteric Gamma Nail 
(TGN) Versus Gamma3 Nail (G3N) - A Review of 217 Cases. Open Orthop J. 2016 Aug 10;10:389-395.

16. Zhang L, Shen J, Chen S, Wu Z, Huang Z, He S, Ying Z, Liu D, Wang Y. Treatment of unstable intertrochanteric femoral fractures with locking gamma nail (LGN): A retrospective cohort study. Int J Surg. 2016 Feb;26:12-7

17. Parker MJ, Pryor GA; Gamma versus DHS nailing for extracapsular femoral fractures: meta-analysis of ten randomised trials. Int Orthop. 1996;20(3):163-8.

18. Schipper IB , Steyerberg EW, Castelein RM, van der Heijden FH, den Hoed PT, Kerver AJ,
15. Huang $H$, Xin J, Ma B. Analysis of complications of intertrochanteric fracture treated with Gamma 3 intramedullary nail. Int $\mathbf{J}$ Clin Exp Med. 2014 Oct 15;7(10):3687-93 van Vugt $A B$. Treatment of unstable trochanteric fractures. Randomised comparison of the gamma nail and the proximal femoral nail. J Bone Joint Surg Br. 2004 Jan;86(1):8694.

19. Bernard ER, Raphael OA, Temilolu O, Bola O. Short gamma nail fixation for intertrochanteric fractures in the elderly. Eur J Orthop Surg Traumatol 2011; 21:275-279.

To cite this article: Mohammed Al Zahhar, Emad Esmat, Amr El Gazzar, Basem Arnaout. Fixation of Unstable Peritrochanteric Fractures of Femur Using Gamma Nail. BMFJ 2021:38 (orthopedic surgery): 86-96. DOI:10.21608/bmfj.2021.15026.1023 